\title{
ㄷำ1
}

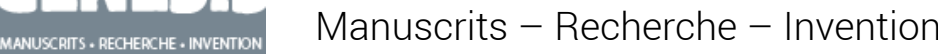

$31 \mid 2010$

Composer

\section{La « Genèse » de Salammbô}

\section{Agnès Bouvier}

\section{OpenEdition}

\section{Journals}

Édition électronique

URL : http://journals.openedition.org/genesis/371

DOI : 10.4000/genesis.371

ISSN : 2268-1590

\section{Éditeur :}

Presses universitaires de Paris Sorbonne (PUPS), Société internationale de génétique artistique littéraire et scientifique (SIGALES)

\section{Édition imprimée}

Date de publication : 10 octobre 2010

Pagination : 109-126

ISBN : 978-2-84050-711-6

ISSN : $1167-5101$

\section{Référence électronique}

Agnès Bouvier, «La «Genèse » de Salammbô », Genesis [En ligne], 31 | 2010, mis en ligne le 24

septembre 2012, consulté le 30 avril 2019. URL : http://journals.openedition.org/genesis/371 ; DOI :

10.4000/genesis.371 


\section{La «Genèse » de Salammbô}

Agnès Bouvier

$\mathrm{L}$

A Littérature SACRÉE DE CARThage a disparu.

Toute ? Non. «Un seul lambeau a surnagé1 », mais phénicien. Il faut entendre les lamentations des orientalistes du XIX ${ }^{\mathrm{e}}$ siècle pour comprendre le regret et la passion qu'a pu susciter l'existence des quelques pages à peu près authentiquement phéniciennes parvenues jusqu'à nous et pour mesurer, dans ce contexte, l'apport du travail de Flaubert dans Salammbô. Le regret s'exprime sous la forme d'un paradoxe si abondamment répété qu'il se constitue en topos de la philologie contemporaine : «le peuple auquel presque toutes les nations civilisées doivent l'écriture alphabétique ne nous a pas laissé de monument de littérature 2 ». La passion des savants se déchaîne dans un déferlement de critiques, contre-critiques, polémiques et mises au point contradictoires sur l'authenticité et l'interprétation du texte phénicien. Salammbô s'écrit au milieu des éclats de cette autre bataille, aujourd'hui oubliée.

De quoi s'agit-il ? Au commencement du $\mathrm{II}^{\mathrm{e}}$ siècle après J.-C., un certain Philon de Byblos, originaire de Tyr, écrit en grec une Histoire phénicienne qu'il prétend avoir traduite d'un texte phénicien dont l'auteur appartiendrait à la plus haute Antiquité (il serait le contemporain de Moïse !), Sanchoniathon. Le livre de Philon a disparu, mais quelques fragments en ont été conservés sous forme de citations dans la Préparation évangélique d'Eusèbe, évêque de Césarée au début du IVe siècle. Ces fragments concernent la partie cosmogonique et théogonique de l'ouvrage. On y trouve une «Genèse » dont les similitudes et les différences par rapport au Pentateuque ont excité l'intérêt des exégètes depuis le XVIe siècle jusqu'à ce moment du XIX ${ }^{\mathrm{e}}$ siècle où la tension croissante entre les savoirs et les croyances place la «contreGenèse » phénicienne au cœur de la critique rationnelle des Antiquités bibliques. Les traductions rivales et les commentaires se multiplient ${ }^{3}$. Flaubert, ultime traducteur, fait énoncer cette Genèse païenne à Shahabarim dans le chapitre III de Salammbô. Ce chapitre, intitulé «Salammbô », présente le portrait mystique de l'héroïne tout entière attachée à Tanit et dévorée du besoin d'en connaître les mystères. Shahabarim, le grand'prêtre de la déesse, cède partiellement aux instances de Salammbô et, «pour la distraire par des perspectives plus hautes », lui conte «le secret des origines 4 ». Voici le passage de Flaubert dans sa version définitive :

Avant les Dieux, les ténèbres étaient seules, et un souffle flottait, lourd et indistinct comme la conscience d'un homme dans un rêve. Il se contracta, créant le Désir et la Nue, et du Désir et de la Nue sortit la Matière primitive. C'était une eau bourbeuse, noire, glacée, profonde. Elle enfermait des monstres insensibles, parties incohérentes des formes à naître et qui sont peintes sur la paroi des sanctuaires.

Puis la Matière se condensa. Elle devint un œuf. Il se rompit. Une moitié forma la terre, l'autre le firmament. Le soleil, la lune, les vents, les nuages parurent ; et, au fracas de la foudre, les animaux intelligents s'éveillèrent. Alors Eschmoûn se déroula dans la sphère étoilée ; Khamon rayonna dans le

1. Ernest Renan, Mémoire sur l'origine et le caractère véritable de l' histoire phénicienne qui porte le nom de Sanchoniathon, Paris, Imprimerie impériale, 1858, in-4, p. 1 [désormais Sanchoniathon].

2. Ibid., p. 3

3. Outre les traductions de Creuzer et de Renan dont nous faisons état dans le dossier génétique, on doit signaler celles de Migne et Séguier, que nous avons consultées mais que nous ne donnons pas pour ne pas alourdir l'ensemble. Il faut mentionner aussi, dans ce très riche corpus, l'existence d'un curieux ouvrage : Friedrich Wagenfeld, Analyse des neuf livres de la chronique de Sanchoniaton, traduit de l'allemand par P. Lebas, Paris, 1836. Ce livre, que Flaubert fait figurer dans la bibliographie qu'il compose en 1862 pour répondre aux objections de G. Froehner, prétend donner la traduction intégrale de l'Histoire phénicienne à partir d'un manuscrit original « retrouvé » au Portugal. La mystification sera très vite dénoncée.

4. Gustave Flaubert, Salammbô, éd. Gisèle Séginger, Paris, GF Flammarion, 2001, p. 111. 
soleil ; Melkarth, avec ses bras, le poussa derrière Gadès ; les Kabyrim descendirent sous les volcans, et Rabbetna, telle qu'une nourrice, se pencha sur le monde, versant sa lumière comme un lait et sa nuit comme un manteau ${ }^{5}$.

Le travail de Flaubert sur les sources peut s'évaluer phrase à phrase ou, si l'on veut, verset après verset. On se propose d'en étudier ainsi la production depuis la source grecque jusqu'au dernier état du brouillon manuscrit de Salammbô. Signalons que, en l'absence de notes de lectures de Flaubert sur le sujet (toutes n'ont pas été retrouvées et il en reste un grand nombre dans des collections privées), la reconstitution du processus de création ne peut être que conjecturale pour ce texte. Les lectures de Flaubert sont cependant suffisamment connues pour justifier une telle démarche.

Le texte grec (A) est présenté dans sa traduction récente, celle de Jean Sirinelli et Édouard des Places ${ }^{6}$. La version de Creuzer retraduite par Guignaut (B) dans les Religions de l'Antiquité est la source principale de Flaubert, qui possède le livre depuis au moins aussi longtemps que la veillée funèbre d'Alfred Le Poittevin7. Les notes du livre quatrième, rédigées par Alfred Maury que Flaubert connaissait bien, ont été consultées par le romancier avec une attention particulière. Il importe enfin de mentionner la contribution de Renan (C) au renouvellement de la traduction des éléments de cosmogonie phénicienne ; on sera frappé de la modernité de la version qu'il publie en 1858 dans son Mémoire sur l'origine et le caractère véritable de l'histoire phénicienne qui porte le nom de Sanchoniathon. Rappelons que la rencontre entre Flaubert et Renan a lieu quelques mois plus tard. Au contact du savant, l'écrivain a pu rassembler toutes les données nécessaires pour, à son tour, interpréter et revivifier la "Genèse » antique, écrire non pas sur l'Antiquité mais depuis ses textes fondateurs poétiquement restitués dans leur vérité originelle. Car c'est bien de cela qu'il s'agit pour Flaubert : forger, non pas une imitation, mais un équivalent de texte ancien - être l'autre oublié qui parle.

Pour plus de clarté, nous numérotons chaque segment du passage définitif en chiffres romains, de I à VI. Puis nous donnons à chaque fois les sources, de $\mathrm{A}$ à $\mathrm{C}$, et enfin les extraits de brouillons correspondants (D), de 1 à 4 ou 1 à 5. Les brouillons concernés de Flaubert figurent sur les folios 291 à $323 \mathrm{v}^{\circ}$ du manuscrit N.a.fr. 23658 de la Bibliothèque nationale de France (voir fig. 1 et 2). Ils sont présentés dans une transcription diplomatique allégée de certaines variantes non pertinentes pour notre propos.

5. Ibid., p. 110. Le dernier segment de la dernière phrase, de « et Rabbetna » à la fin, réclamerait une étude à part, qui sera publiée ultérieurement. Nous le gardons ici pour ne pas tronquer le texte de Flaubert.

6. Eusèbe, La Préparation évangélique, Paris, Éditions du Cerf, 1974, p. $184-185$.

7. G.F. Creuzer, Religions de l'Antiquité considérées principalement dans leurs formes symboliques et mythologiques, t. IV, trad. et complété par J.D. Guignant, Paris, s.n., 1841 


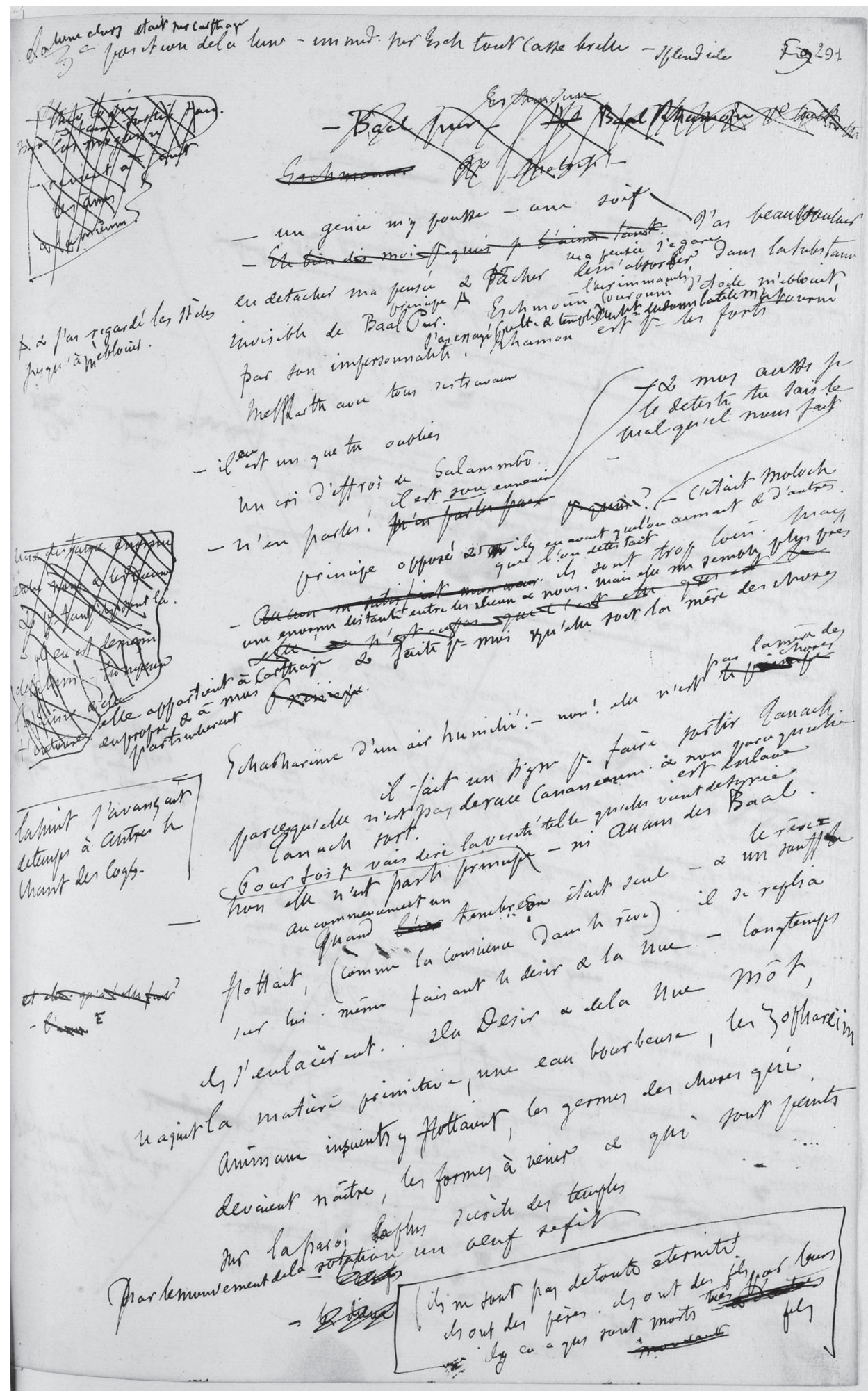

Fig. 1 : Gustave Flaubert, 


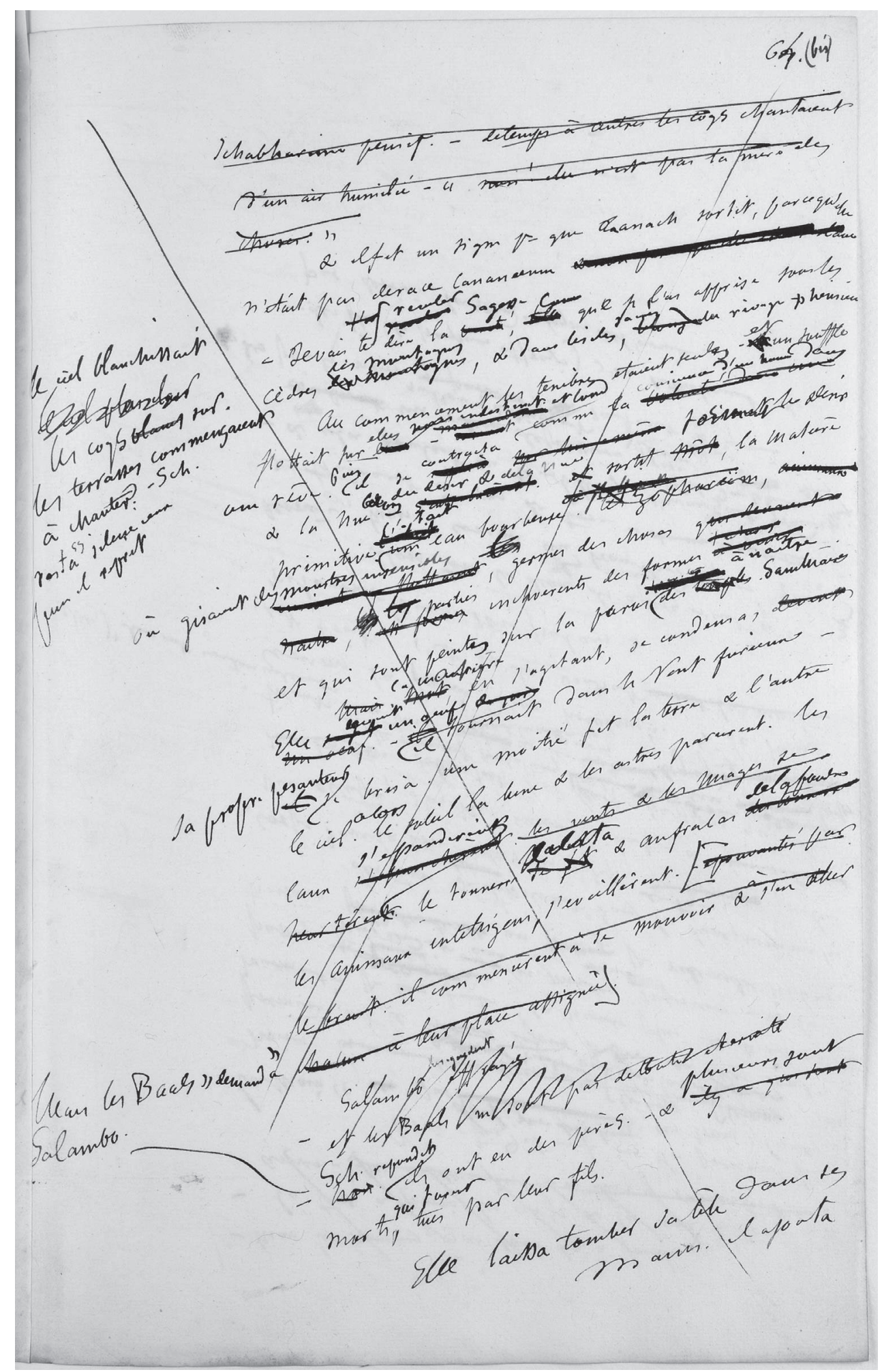




\section{Corpus}

\section{I}

Avant les dieux, les ténèbres étaient seules, et un souffle flottait, lourd et indistinct comme la conscience d'un homme dans un rêve.

\section{A (Eusèbe)}

Il place à l'origine de l'Univers un air opaque et venteux ou un souffle d'air opaque, et le chaos bourbeux, ténébreux. Ces éléments étaient infinis et restèrent sans limite pendant une longue durée de temps.

\section{B (Creuzer)}

a. Chez Eusèbe, le Souffle de l'esprit ou le Vent primitif, et la Nuit primitive, figurent comme principes des choses.

b. note 1, p. 13 :

Dans le fragment original, ce n'est point par ce couple mythique que débute la cosmogonie de Sanchoniathon, mais par un air ténébreux pareil à un souffle, à un esprit, et par un chaos confus, enveloppé de ténèbres. Ces deux principes existent de tout temps dans l'espace infini.

c. notes du livre quatrième :

Sanchoniathon, d'après Taaut, c'est-à-dire d'après les livres sacrés des Phéniciens, ouvrages de leurs prêtres, pose comme le principe de l'univers un Air ténébreux et plein du souffle (de l'esprit), ou bien le Souffle d'un air ténébreux et un Chaos confus enveloppé d'une obscurité profonde. L'un et l'autre étaient infinis et sans limites dans le cours des âges.

\section{C (Renan)}

Au commencement était le Chaos, et le Chaos était ténébreux et troublé, et le Souffle planait sur le Chaos.

Et le Chaos n'avait pas de fin, et il fut ainsi durant des siècles de siècles

\section{D (brouillons de Flaubert)}

\section{1}

- No`elle `est pas le pri` cipe - `i aucu` des Baal.

$$
\text { Au comme`ceme ` } t \text { ' } \mathrm{\prime} \text { ' } \mathrm{s} \text { le rêve }
$$

Qua`d l'air te` ebremx était seul - \& u` souffle

flottait, (comme la co`scie`ce da`s le rêve).

(fo 291)

\section{2}

Au comme` ceme`t les te`èbres etaie`t seules - et u`souffle mais i disti` ct et lourd

elles mais [illis.] co`scie`ce d'u` homme da`s

flottait sur [illis.] - [illis.] comme la volo'té da's ẗ

u’ rêve.

$$
\left(f^{\circ} 323 v^{\circ}\right)
$$

\section{3}

d'ab

- Au comme` ceme` t, les te èbres etaie`t seules - \& u` souffle mais

flottait swrelles, i` disti` ct \& lourd comme la co` scie`ce d'u’ homme da's u’ rêve.

$\left(f^{\circ} 318\right)$

- Au comme` ceme` t les Te`èbres etaie` t seules. et u` Souffle flottait, lourd \& i` disti`ct comme la co`scie`ce d'u` homme da`s u’ rêve.

$\left(\mathbf{f}^{\circ} 306\right)$ 
Il se contracta, créant le Désir et la Nue, et du Désir et de la Nue sortit la Matière primitive. C'était une eau bourbeuse, noire, glacée, profonde.

\section{A (Eusèbe)}

Mais lorsque, dit-il, le souffle se prit d'amour pour ses propres principes et que se produisit un mélange, on appela cette combinaison le désir. C'est là le principe de la création de toute chose. Mais lui-même ne connaissait pas sa propre création. De la combinaison du souffle avec lui-même naquit Môt. Selon certains, c'est le limon ; selon d'autres, la putréfaction d'un mélange aqueux.

\section{B (Creuzer)}

a. Vient ensuite le Désir ou l'Amour, qui n'est autre que l'esprit se repliant sur lui-même, et la cause de la création. Le fruit de cette union mystérieuse est $M o ̂ t$, ou la matière première, refermant dans son sein les semences de tous les êtres.

b. les Sidoniens supposent antérieurs à toutes choses le Temps, le Désir et la Nue. Le Désir et la Nue s'étant unis l'un à l'autre, comme les deux principes par excellence, de leur union naquirent l'Air et la Brise. Par l'Air, ajoute l'interprète, ils désignent l'intelligible pur ; par la Brise, le prototype de la vie animale, qu'elle met en mouvement.

Mais quand le Souffle ou l'Esprit (pneuma), ajoute-t-il, se fut épris de ses propres principes, et qu'ils se furent unis entre eux, cette union fut appelée l'Amour (Poqos), et telle fut l'origine de la création de l'univers. Mais l'Esprit ne connaissait pas sa propre création, et de l'union qu'il contracta naquit Môt, que les uns interprètent par le limon, les autres par une eau bourbeuse en putréfaction.

\section{C (Renan)}

Et le Souffle aima ses propres principes, et il se fit un mélange, et ce mélange fut appelé Désir.

Et le Désir fut le principe de la création de tout, et le Souffle ne connaissait pas sa propre création.[...] Et le Souffle et le Chaos se mêlèrent, et Môt (élément boueux) naquit.

\section{1}

il se replia

sur lui-même faisant le desir \& la nue - longtemps ils s'enlacèrent. Du Desir \& de la nue môt, naquit la matière primitive, une eau bourbeuse,

$\left(\mathbf{f}^{\circ} 291\right)$

\section{2}

Puis contracta

il se replia sur lui même faisant/formant le Desir alors du desir \& de la nue

\& la nue. - s'entrelacèrent. et sortit môt, la matière c'etait

e'etait

primitive une eau bourbeuse.

$\left(\mathbf{f}^{\circ} 323 \mathbf{v}^{\circ}\right)$

3

Puis it se contractant

isolant

forma le desir \& la nue - \& du Desir \& de la nue sortit la Matière Primitive. c'etait une profondes.

eau bourbeuse, noire et glacée

$\left(\mathbf{f}^{\circ} 318\right)$

\section{4}

il se contracta, creant

le Desir \& la nue. - \& du Desir \& de la nue, sortit la matiere primitive. C'etait une eau bourbeuse, noire, glacée, profonde.

$\left(\mathbf{f}^{\circ} 306\right)$ 


\section{III}

Elle enfermait des monstres insensibles, parties incohérentes des formes à naître et qui sont peintes sur la paroi des sanctuaires.

\section{A (Eusèbe)}

De là provint toute semence de création et la genèse de l'Univers. Il y avait des animaux dépourvus de sentiment, de qui naquirent des êtres doués de l'esprit, et ils furent appelés Zophasemin, c'est-à-dire contemplateurs du ciel.

\section{B (Creuzer)}

a. De là certains animaux d'abord dépourvus de sentiment b. note 1, p. 13 :

renfermant dans son sein les semences de tous les êtres.

c. le prototype de la vie animale, qu'elle met en mouvement.

C'est d'elle (de cette matière première) que procéda toute semence de création et la génération du monde entier. Il y avait certains animaux dépourvus de sentiment, desquels naquirent des animaux doués d'intelligence ; et ils furent appelés Zophasemin, c'est-à-dire contemplateurs du ciel

\section{C (Renan)}

Et de Môt sortit toute semence de création, et Môt fut le père de toute chose.

[...] Et il y avait des êtres vivants privés de sentiment, et de ces êtres vivants naquirent des êtres intelligents, et on les appela Zophésamin.

\section{D (brouillons de Flaubert)}

les zophareïm

animaux inscients y flottaient, les germes des choses qui devaient naitre, les formes à venir \& qui sont peints sur la paroi la plus secrète des temples

(fo 291)

2

\& flottaient

tes zophareim, animaux

gisaient des monstres insensibles les

[illis.] y flottaient, germes des choses quidevaient

parties futures

naitre, et formes inchoerentes des formes à venir

à naitre

et qui sont peintes sur la paroi des semples. sanctuaires

$\left(\mathbf{f}^{\circ} 323 \mathrm{v}^{\circ}\right)$

\section{3}

Elle enfermait

origine des choses.

des monstres insensibles, germes des choses, parties incoherentes à naitre à naitre

des formes futmes \& qui sont peintes sur la paroi des futures

sanctuaires.

$\left(\mathbf{f}^{\circ} 318\right)$

4

Elle enfermait des monstres

insensibles parties incoherentes des formes futures \& qui sont peintes sur la paroi des sanctuaires.

(fo 306) 
IV

Puis la Matière se condensa. Elle devint un œuf. Il se rompit. Une moitié forma la terre, l'autre le firmament.

\section{A (Eusèbe)}

Ils furent façonnés à la ressemblance d'un œuf

\section{B (Creuzer)}

II, 3, notes du livre quatrième, note 3, p. 858-861 :

- quant à l'œuf, c'est le Ciel. On dit, en effet, que cet œuf s'étant brisé en deux moitiés, une de ces moitiés forma le ciel, et l'autre la terre.

- et ils reçurent la figure d'un œuf

\section{C (Renan)}

Et Môt avait la forme d'un œuf

\section{D (brouillons de Flaubert)}

\section{1}

Par le mouvement de la rotation un œuf se fit

$$
\text { - œuf }
$$

Il tournait dans le vent furieux, à force de tourner L'œuf se brisa en deux - une moitié la terre - l'autre le ciel -

$$
\text { (f }{ }^{\text {ps }} 291 \text { et 293) }
$$

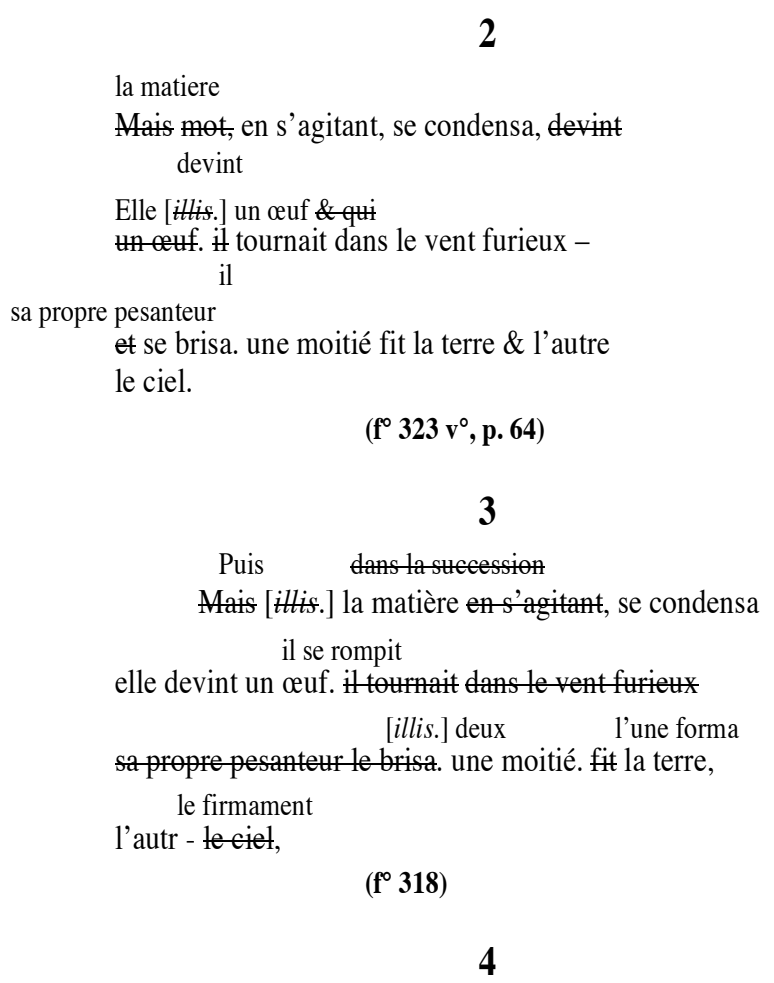

Puis la matière se condensa. Elle devint un œuf. moitié

il se rompit. en deux moitiés. L'une forma la Terre, l'autre le Firmament.

$\left(f^{\circ} 306\right)$ 


\section{V}

Le soleil, la lune, les vents, les nuages parurent ; et, au fracas de la foudre, les animaux intelligents s'éveillèrent.

\section{A (Eusèbe)}

et Môt jeta ses feux, comme aussi le soleil, la lune, les étoiles et les grands astres. » Voilà à peu près leur cosmogonie, prélude manifeste à l'athéisme. Voyons ensuite comment, selon lui encore, eut lieu la génération des animaux. Il s'exprime ainsi : «Et l'air s'étant mis à flamboyer, l'embrasement agissant sur la terre et la mer provoqua des vents, des nuages, des chutes et des déversements considérables d'eaux célestes. Une fois que, à cause de la chaleur solaire, ces éléments eurent été séparés, qu'ils eurent été écartés de leur emplacement propre, qu'ils se furent entrechoqués, alors se produisirent tonnerre et éclairs et, au fracas du tonnerre, les animaux doués d'intelligence et dont il a été parlé se réveillèrent ; ils furent épouvantés par le vacarme, et, mâles comme femelles, commencèrent à se mouvoir sur la terre et dans la mer.

\section{B (Creuzer)}

a. de là le soleil, la lune et les étoiles. Le Souffle primitif et la Nuit enfantèrent Æon et Protogonos (la durée et le premier-né), qui mirent au jour Genos et Genea (genre et race). Vinrent ensuite la lumière, le feu et la flamme, de qui à leur tour le Casius, le Liban et l'Anti-Liban. Après bien des générations parurent Sydyk et les Cabires. On nous parle encore des éclairs et des tonnerres, dont le fracas épouvantable réveilla les animaux qui commencèrent à se mouvoir dans la mer et sur la terre, tant mâles que femelles. b. et du sein de Môt resplendirent le soleil et la lune, les étoiles et les grands astres (les constellations). L'air s'étant illuminé, par l'embrasement de la mer et de la terre se formèrent les vents et les nuages, puis vinrent d'immenses épanchements des eaux célestes tombant avec impétuosité. Et ces choses ayant été ainsi séparées et déplacées par les feux du soleil, et s'étant de nouveau rencontrées dans l'air et violemment heurtées, le tonnerre et les éclairs se firent ; et au fracas du tonnerre les animaux intelligents décrits plus haut s'éveillèrent, et ils furent épouvantés par le bruit, et ils commencèrent à se mouvoir sur la terre et dans la mer, tant mâles que femelles.

\section{C (Renan)}

Et le soleil, et les étoiles, et les grandes constellations brillèrent.

Et il y avait des êtres vivants privés de sentiment, et de ces êtres vivants naquirent des êtres intelligents, et on les appela Zophésamin.

Et les hommes, mâles et femelles, commencèrent à se mouvoir.

\section{D (brouillons de Flaubert) 1}

le soleil la lune $\&$ les astres parurent - les eaux s'epanchèrent, les vents \& les nuages se heurterent le tonnerre se fit. $-\&$ au fracas du tonnerre les animaux intelligents s'eveillèrent - \& épouvantés par le bruit il commencèrent à se mouvoir et à s'en aller chacun dans à leur place $\&$ dans leur place assignée

(f' 293)

\section{2}

alors

le ciel. le soleil la lune \& les astres parurent. les s'epandirent

eaux s'epanchèrent. les vents \& les nuages se

eclata de la foudre heurtèrent le tonnerre se fit $\&$ au fracas du tonnerre les animaux intelligens, s'eveillèrent. epouvantés par le bruit. il commencèrent à se mouvoir \& à s'en aller chacun à leur place assignée.

$\left(f^{\circ} 323 v^{\circ}\right)$

\section{3}

le soleil, la lune les vents les nuages les astres parurent, les cieux s'epandirent alors

te tonnerre eclata \& au fracas de la foudre, les animaux intelligents s'eveillèrent.

$\left(\mathbf{f}^{\circ} 318\right)$

\section{4}

alors le soleil la lune, les vents les nuages parurent, \& au fracas de la foudre les animaux intelligents s'eveillèrent.

(f ${ }^{\circ}$ 306) 
VI

Alors Eschmoûn se déroula dans la sphère étoilée ; Khamon rayonna dans le soleil ; Melkharth, avec ses bras, le poussa derrière Gadès ; les Kabirim descendirent sous les volcans,

\section{A (Creuzer)}

Ici encore, comme en Égypte, de nombreuses divinités sortent de la sphère théogonique pour recevoir dans le culte public les hommages des mortels et prendre possession des temples. Malheureusement les noms qu'on leur donne sont en grande partie grecs. Uranus (le ciel) a de sa sœur Gê (la terre), Ilus ou Cronus, puis le Bétyle, puis Dagon, appelé encore Siton, puis Atlas. Uranus veut faire périr ses enfants, mais Cronus le détrône avec le secours d'Hermès, son fidèle conseiller, et d'Athène ou Minerve. Cronus prend successivement pour femmes Astarté, Rhéa, Dioné, ses sœurs... Un fils naturel d'Uranus, par conséquent frère consanguin de Cronus, met au jour Melkarth (Hercule)... Il bâtit la ville de Byblus, qu'il donne en présent à sa femme et sœur Baaltis, la même que Dioné.

\section{D (brouillons de Flaubert)}

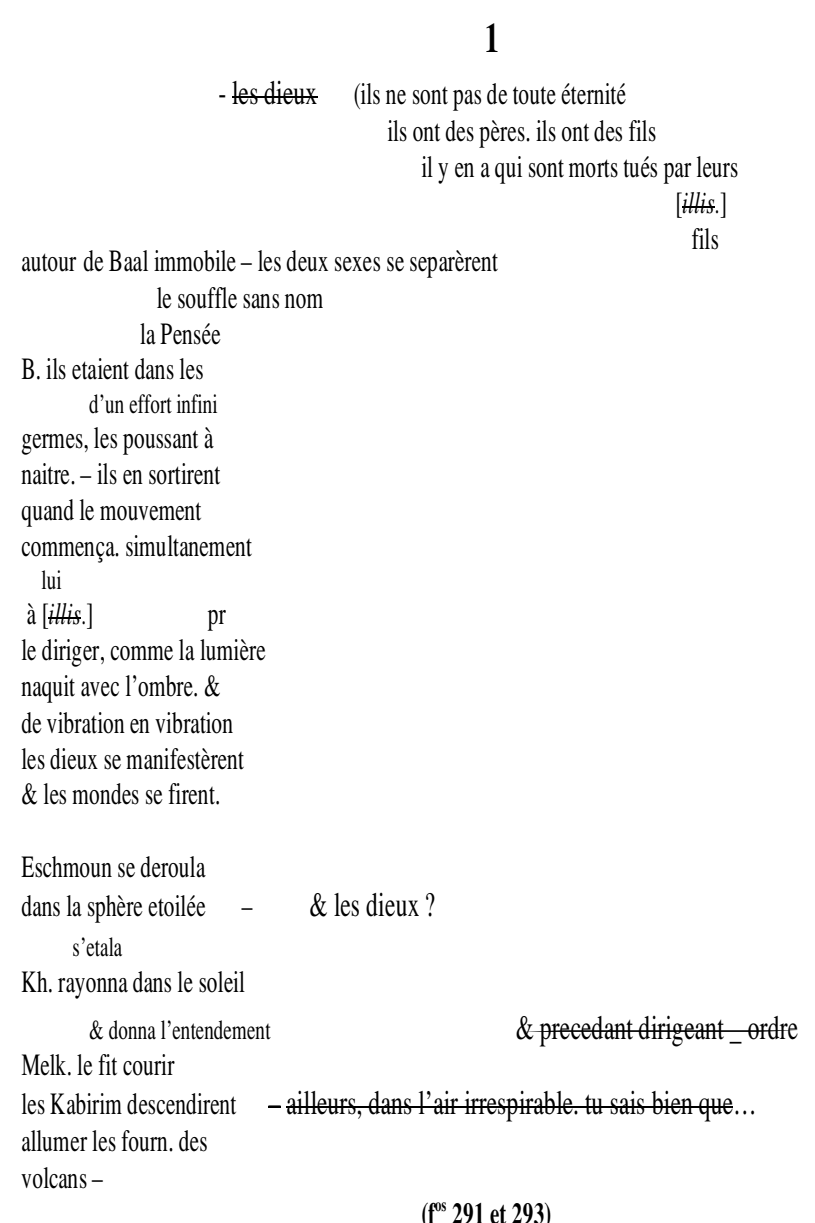

Salambôeffrayée

- et les Baals ne sont pas de toute eternité

\author{
Sch. repondit plusieurs son \\ - non. ils ont eu des pères. - \& ily a qui sont \\ qui furent \\ morts, tués par leur fils. \\ Elle laissa tomber sa tête dans ses \\ mains. il ajouta \\ $\left(f^{\circ} 323 v^{\circ}\right)$
}


- où etaient-ils"

ils etaient au sein de lui-même, - du Baal immobile. dans le souffle sans

$$
\& \text { cachés, }
$$

nom, dams laPensée, - sous les germes de leurs propres formes -

$$
\text { en sortir }
$$

faisant un s'effort/cant constant pr briser l'enveloppe.quandleavec le mouvement commença, its commencèrentet la Vie partut

en même temps qu'eux, comme l'ombre \& la Lumière. les

mondes se firent \& les Dieux furent. quiles dirigent.

\&alors Eschmoun se deroula dans la sphère etoilée. Khamon

$$
\text { avec ses bras }
$$

rayonna dans le soleil. Melkarth le poussa par delà

$$
\text { dans les }
$$

l'occident. les Kabirim descendirent, allumer les fourneaux des volcans,

$$
\left(f^{\circ} 314 r^{\circ}\right)
$$

\section{4}

et

- "Mais les Baals." demanda Salambo

Schabharim repondit awec un sourire de dedain

- ils onteu des pères. \& plusieurs sont morts qui furent tués parleurs fills."

Elle laissamber satête dans ses mains.
celluiqu'on represente
par une stele d'or \& une
encore

- ils etaient au sein du Baal immobile dans les profondeurs cachés

leurs propres

de l'esprit \& cachés sous les germes de leur propre forme -$$
\text { avec }
$$

perment

quand le mouvement commença les dieux naquirent.

et de suite alors Eschmoun se deroula dans la sphère etoilée, \& avec

avec

elle Khamon rayonna dans le soleil, melkarth de ses bras le poussa derrière Gades, les Kabirim descendirent sous les volcans

dans

\section{5}

- et les Baals,?" demanda Salambô

- Ils etaient au sein du Baal - immobile, cachés dans

les profondeurs de l'esprit, cachés sous leurs propres

$$
\text { ils }
$$

quand le mouvement commença, les dieux naquirent. et alors Eschmoûn se deroula dans la sphère étoilée,

Khamon rayonna dans le soleil, Melkharth avec ses bras

le poussa derrière Gadis, les Kabirim descendirent dams les volcans

$\left(\mathbf{f}^{\circ} 306\right)$ 


\section{Pistes interprétatives}

La seule mise en présence de ces différents états permettrait de comprendre intuitivement le sens des choix opérés par Flaubert. Quelques remarques peuvent cependant avoir leur utilité, qui aborderont successivement les enjeux philologiques, religieux et scientifiques de la retraduction de la «Genèse » phénicienne.

\section{Retour philologique en Phénicie}

Le travail du romancier sur le texte source consiste d'abord à le dégager de son «triple voile » d'intermédiaires helléniques, chrétiens et modernes pour le rendre à son origine « chananéenne ».

Le problème qui se pose d'emblée est en effet celui de la transmission grecque du texte phénicien, à la fois conservé et dénaturé par sa traduction-trahison. Flaubert est conscient de cette difficulté majeure d'accès aux littératures orientales anciennes. La phrase de la lettre à Sainte-Beuve sur le Périple d'Hannon « échenillé par un $\mathrm{Grec}^{8}$ » montre qu'il suit la leçon de ses principaux interlocuteurs pour les questions philologiques relatives à la Phénicie : Renan et Maury via Creuzer : « c'est de la quatrième main seulement que nous tenons les lambeaux de la mythologie phénicienne. On conçoit qu'à une distance d'époques aussi considérable, le texte du Phénicien Sanchoniathon avait dû être peu fidèlement rendu, peut-être même arbitrairement modifié et systématiquement interprété par son traducteur grec $^{9} »$. Les hellénismes des traducteurs successifs affectent principalement les noms des dieux dans la partie théogonique de l'Histoire phénicienne. Creuzer en fait la remarque : "Malheureusement les noms qu'on leur donne sont en grande partie grecs ${ }^{10}$. » Flaubert opère, dans la perspective de re-sémitisation du texte qui est la sienne, une «translation » inversée de l'onomastique, substituant par exemple «Melkarth » à Hercule. Il est familier de ce jeu de correspondances sur lesquelles il fonde une grande partie de ce qu'il appelle ses « inductions » sur Carthage.

Mais le lexique phénicien, on s'en aperçoit en comparant les différentes versions manuscrites du passage, sert avant tout à Flaubert de pilotis sur lequel il (re)construit la langue de Carthage. Ainsi du mot «môt », conservé jusqu'à la deuxième version manuscrite et passé de la majuscule à la minuscule, le nom propre devenant, à la lettre, un « mot »: résidu phénicien irréductible à la traduction, insoluble dans le grec et le français, innommable sauf à en donner des périphrases. Philon, Eusèbe et tous les traducteurs modernes donnent un état de la question sur le sens de « Môt »: « Selon certains, c'est le limon ; selon d'autres, la putréfaction d'un mélange aqueux »(Eusèbe) ; «que les uns interprètent par le limon, les autres par une eau bourbeuse en putréfaction » (Creuzer). Flaubert règle la discussion en convertissant les hypothèses différentes en vérité unique : «C'était une eau bourbeuse, noire, glacée, profonde. » Il procède à la fusion des interprétations en unissant les sèmes « aqueux » et « limon » dans la formule « eau bourbeuse », et se livre ainsi à un travail de retraduction en direction de la source du mot, vers son unité sémantique originelle.

L'autre mot phénicien dont la présence est maintenue jusqu'à la deuxième version est «zophareïm ». Les traducteurs donnent «zophasemin », à l'exception de Renan qui transcrit «zophésamin » et voit dans le caractère typiquement sémitique du terme (« la physionomie sémitique de zofhsamhn n'est point méconnaissable11 ») une preuve de l'authenticité de l'Histoire phénicienne. Pourquoi Flaubert a-t-il écrit «zophareïm»? On peut penser à une erreur de copie qui se serait répercutée dans les « notes de notes » et les brouillons successifs, occasionnée par la tendance de Flaubert, remarquée par tous ceux qui lisent ses manuscrits, à inverser des lettres ou des syllabes dans les noms propres ou les mots étrangers. On peut aussi préférer l'hypothèse d'une variante « carthaginoise » produite par Flaubert sur la base du mot phénicien transcrit en grec et voir dans cette modification la mise en œuvre de l'imagination linguistique du romancier qui invente pour Salammbô une musique punique. La création la plus originale de Flaubert dans ce domaine est, on le sait, le mot «zaïmph ». Le mot phénicien sert de matériau

8. Flaubert, Correspondance, Paris, Gallimard, coll. « Bibliothèque de la Pléiade », t. III, 1991, p. 275.

9. Creuzer, Religions de l'Antiquité, op. cit., p. 10.

10. Ibid., p. 14-15.

11. Renan, Sanchoniathon, op. cit., p. 15. 
pour la construction d'une langue propre à Carthage que Flaubert restaure par l'érudition et la fantaisie puis efface pour écrire son roman « traduit du Carthaginois 12 ».

Le texte source pose également la question de l'énonciation dans la mesure où il se présente à Flaubert comme une parole rapportée, aussi bien dans le texte grec que dans les traductions contemporaines. La « Genèse phénicienne »n'est jamais énoncée comme vérité mais comme croyance. La modalisation marque la distance entre la position d'Eusèbe, évêque chrétien, et celle du païen Philon, distance encore élargie par les traducteurs catholiques français du XIXe siècle. Creuzer puis Guignaut creusent davantage l'écart en proposant d'emblée un commentaire : " Dans le fragment original, ce n'est point par ce couple mythique que débute la cosmogonie de Sanchoniathon, mais par un air ténébreux pareil à un souffle, à un esprit, et par un chaos confus, enveloppé de ténèbres. » Le correctif apporté par la note ajoute un niveau supplémentaire de médiation. L'original ne se distingue plus de l'exégèse dont il est l'objet. Tout entier livré à la critique philologique, il est parasité par les gloses. Le romancier a donc pour tâche de retrouver par induction le texte original gauchi par le zèle des interprètes et le restituer à son énonciateur indigène, le prêtre « chananéen ». En reconstituant par la fiction la situation d'énonciation propre au texte sacré, depuis la caste sacerdotale représentée par Shahabarim jusqu'à l'impétrant figuré par Salammbô, Flaubert réalise l'incarnation de la parole mythique dans les personnages en présence. Du coup, la cosmogonie est restituée à sa sphère de croyance, émise et reçue comme vraie dans le cadre de référence interne du roman.

Parallèlement, Flaubert cherche à retrouver la densité qu'il imagine devoir être celle du texte phénicien en le débarrassant des longueurs, des délayages, des redites, de toutes les scories déposées par les compilateurs successifs. Le processus qui aboutit à la séquence « au fracas de la foudre, les animaux intelligents s'éveillèrent », est révélateur de ce travail de densification du texte source. Flaubert suit d'abord Creuzer (chapitre de Maury), qu'il paraphrase presque terme pour terme dans sa première version. En dépit de quelques ellipses, des mouvements entiers sont directement repris. Les versions suivantes procèdent à la contraction du texte par effacement des liens de causalité. L'explication scientifique (les nuages naissent de l'évaporation de l'eau) est éliminée pour créer un effet de surgissement simultané des phénomènes. Les traces de rationalité grecque disparaissent dans la commotion originelle poétiquement retrouvée. La troncation de la phrase permet sa chute sur « éveillèrent » et la fait résonner pour nous comme un vers illuminé de Rimbaud. La violence exercée sur la phrase source lui imprime une convulsion qui la fait jaillir en cataracte (on notera dans le « fracas de la foudre » un cas d'allitération volontairement conservée par Flaubert) et la dégage par la biffure du fatras explicatif occidental. Flaubert fait acte de retraducteur moderne en extrayant le texte du logos pour le rendre aux seuls effets perceptifs, à la physis. Le peplos grec se déchire et laisse apparaître le texte original réinventé dans sa sidérante étrangeté, son obscurité native, sa brièveté, ses marques d'Antiquité, en somme - du moins si l'on en croit les philologues contemporains pour qui ces critères sont déterminants lorsqu'il s'agit d'évaluer le degré d'authenticité d'un texte : «Dans les morceaux les plus anciens il offre un cachet antique, il est bref et parfois obscur ; dans ce qui est plus récent, il règne plus de clarté, et les détails sont plus nombreux ${ }^{13}$. » Afin de fabriquer l'authentique, le romancier a dû convertir ces outils critiques en instruments de création.

\section{Vers une Genèse sans Dieu?}

En réduisant la cosmogonie phénicienne à son noyau originel, Flaubert n'évacue pas pour autant la controverse religieuse qui l'entoure, bien au contraire : il inscrit le débat au cœur même du texte ainsi retraduit en soulignant son caractère matérialiste. Les choix traductifs qu'il opère vont tous dans la même direction : celle d'une antiGenèse.

Pour mesurer les enjeux du passage qui nous occupe, il faut d'abord comprendre que le problème de l'authenticité de l'Histoire phénicienne, discuté tout au long du XIX ${ }^{\mathrm{e}}$ siècle, questionne directement le statut

12. C'est le titre d'une série de caricatures sur Salammbô publiées par Morel-Retz, dit Stop, dans Le Journal amusant, 10 janvier 1863, p. 4-6. 13. Wagenfeld, Analyse des neuf livres de la chronique de Sanchoniation, op. cit., p. 87. 
de la Genèse biblique. S'il s'agit en effet d'un texte véritablement phénicien, cela remet en cause la singularité de la Bible qui ne serait plus alors qu'une variante de la «mythologie primitive des Sémites 14 ». Si, au contraire, l'Histoire phénicienne est un faux, ce pourrait être une contrefaçon de la Genèse, pervertie sous l'influence d'une philosophie matérialiste et athée15. Quoi qu'il en soit, les échos qui se renvoient d'une tradition à l'autre sont pour le moins troublants et contribuent à relativiser le texte biblique en même temps qu'à en infléchir l'interprétation.

L'intérêt des penseurs rationalistes pour l'Histoire phénicienne provient essentiellement de son caractère matérialiste et athée. Eusèbe le signalait déjà pour s'en indigner : «Sanchoniathon, dont nous allons analyser les écrits, n'attribue pas la divinité au Dieu de l'univers [...] il introduit évidemment l'athéisme16. » Guignaut résume ainsi le propos de Philon traduisant Sanchoniathon : «Sapant toute religion par la base, il montrait dans les fables phéniciennes et égyptiennes [...] une suite de récits historiques remontant à l'origine du genre humain et du monde lui-même, issus l'un et l'autre de principes matériels ${ }^{17}$. » La cosmogonie récrite par Flaubert a donc la particularité d'être une création sans créateur.

«Avant les dieux, les ténèbres étaient seules, et un souffle flottait, lourd et indistinct comme la conscience d'un homme dans un rêve »: la version de Flaubert souligne explicitement, en la portant au-devant de la phrase, la position secondaire de l'action divine dans la création du monde. Au folio 291 on trouve : « Non elle n'est pas le principe - ni aucun des Baal. » La négation des dieux se posait de manière encore plus radicale comme le «contre-principe » du monde. «Avant les dieux » contracte un long développement qui introduisait la théogonie et se présentait sous la forme d'un dialogue entre Shahabarim et Salammbô ( $f^{\circ} 323$ ). La révélation de Shahabarim provoquait l'accablement de Salammbô : les dieux ne sont pas à l'origine du monde, ils naissent, se reproduisent et meurent, ce qui signifie qu'à l'origine les dieux ne sont que des hommes. Ce catéchisme à rebours, cette initiation au sacré par la désacralisation permettait à Flaubert d'intégrer dans le roman la critique évhémériste du christianisme et la controverse entre Philon et Eusèbe sur la question de la fabrication des dieux par les hommes.
Dans cette Genèse sans Dieu, seule la matière est « première ». Tous les traducteurs sont formels : il faut comprendre que, dans la cosmogonie phénicienne, la matière est l'origine unique de toute chose, l'esprit ne venant qu'en seconde position. Flaubert, lui, introduit dans sa cosmogonie l'idée d'une concomitance entre le surgissement de la matière et celui de l'esprit. Avec « et un souffle flottait, lourd et indistinct comme la conscience d'un homme dans un rêve », la matérialisation de l'esprit soumis à la pesanteur («lourd ») et l'élévation de la matière («flottait») s'énoncent dans le même souffle. Le paradoxe vient s'articuler autour de la virgule à la fois séparatrice et unifiante : «flottait, lourd ». L'opposition entre la matière et 1 'esprit 18 est résolue par la présence absente du rêveur dont la figure sans contour se déploie et s'anéantit dans le dernier mouvement rythmique de la phrase. Il est question d'une présence indéfinie, sans forme ni étendue. La comparaison porte à faux, le comparant abstrait ( "comme la conscience ») ne permettant pas à l'image de se former : elle donne à voir une présence génésiaque et en même temps l'évacue dans la mesure où il ne s'agit précisément que d'une figure de style. Tout se passe comme s'il y avait quelqu'un, mais il n’y a personne. Dieu comme volonté (f ${ }^{\circ} 323$ ) a disparu, il n'en reste que la trace métaphorique.

La production de cette nouvelle Genèse réagit sur la Genèse du Pentateuque et en questionne la lettre et l'esprit. Un texte si proche de la Bible et si éloigné de son sens reçu introduit nécessairement un trouble interprétatif que l'on peut formuler ainsi : et si la Genèse phénicienne était finalement plus conforme au texte hébreu que ne

14. Renan, Sanchoniathon, op. cit., p. 15.

15. Ibid.

16. Eusèbe, La Préparation évangélique, trad. Migne, op. cit., livre I, chap. IX, p. 522-523.

17. Maury, op. cit., p. 842 .

18. On sait que le dépassement de cette opposition est l'une des préoccupations de Flaubert : «Je suis convaincu que les appétits matériels les plus furieux se formulent insciemment par des élans d'idéalisme, de même que les extravagances charnelles les plus immondes sont engendrées par le désir pur de l'impossible, l'aspiration éthérée de la souveraine joie. Je ne sais (et personne ne sait) ce que veulent dire ces deux mots : âme et corps, où l'une finit, où l'autre commence. Nous sentons des forces et puis c'est tout. Le matérialisme et le spiritualisme pèsent encore trop sur la science de l'homme pour que l'on étudie impartialement tous ces phénomènes » (Correspondance, éd. cit., t. III, p. 16-17). 
le laissent penser les traductions autorisées de la Bible ? Dans ce cas, la cosmogonie de Sanchoniathon donnerait accès à l'essence même de la Genèse biblique rendue à sa dimension matérialiste occultée par des siècles d'exégèse chrétienne. L'hypothèse a pris corps à l'occasion du renouveau des études bibliques depuis le début du siècle et s'appuie sur les traductions modernes du texte fondateur du christianisme. À cet égard, le débat autour du mot « souffle », que Flaubert intègre dans son premier « verset », est d'un grand intérêt. $\pi v \varepsilon v \mu \alpha$, on le sait, signifie aussi bien «le souffle » que «l'esprit». Saint Jérôme avait maintenu l'ambivalence en traduisant le mot hébreu correspondant par le latin « spiritus ». Mais dans Questions hébraïques sur la Genèse, on voit qu'il choisit clairement le sens figuré en disant que « cela n'est pas dit du souffle du monde, comme quelques-uns le pensent, mais de l'Esprit saint, qui est appelé vivificateur de toutes choses dès le commencement 19 ». " Comme quelques-uns le pensent » laisse supposer que l'acception matérielle ou spirituelle du terme était déjà un sujet de controverse au IVe siècle. Samuel Cahen, dans la traduction du Pentateuque qu'il soumet au public en 1831, suit la tradition et propose « esprit divin ». Mais la note dont il assortit le deuxième verset est beaucoup plus audacieuse et infléchit considérablement l'interprétation du mot hébreu :

Ce mot au propre signifie de l'air en mouvement, le vent ; au
figuré l'esprit. Cette métonymie se rencontre dans beaucoup
de langues [...]. En prenant (ce mot) au propre, on pourrait
voir ici un vent violent ; ce serait le troisième élément qui, à
raison de sa légèreté, était au-dessus des deux premiers, la
terre et l'eau. On sait qu'en hébreu le mot divin sert d'ampli-
fication. Ainsi on dit Sam. I, ch. 14 , v. 15 . Une anxiété divine,
Ps. 36, v. 7 . Des montagnes divines, Genèse, ch. 6 , v. 2 .
Des fils divins, pour exprimer une grande anxiété, de hautes
montagnes, de grands hommes, etc. (l'esprit divin) signifierait
donc un vent violent, un grand vent 20 .

«Divin » ne serait alors rien d'autre qu'une hyperbole et Dieu, décidément, une figure de style. Il faut relier ce « grand vent» au dantesque « vent furieux » dans lequel Flaubert fait tourner la matière primitive (IV, versions 1 et 2). La traduction de Cahen lui est familière ${ }^{21}$, ainsi que l'ouvrage où cette note est signalée, le Traité sur l'hétérogénie de son vieil ami Félix-Archimède Pouchet: «S. Cahen pense que le dernier membre de phrase pourrait bien n'exprimer que l'action d'un vent violent qui labourait la surface de l'abîme22 », écrit le naturaliste.

\section{Entre genèse biblique et pensée scientifique}

Le troisième axe de travail du retraducteur moderne est de lire les sources antiques à la lumière des travaux scientifiques les plus récents. Or, la question de la Genèse est un enjeu majeur de la science du vivant à l'époque de Flaubert dans la mesure où elle départage les tenants du «matérialisme » et les autres. Félix Pouchet est l'un de ceux qui cherchent à annexer la Genèse biblique à la conception matérialiste des origines du monde :

Toutes les cosmogonies s'accordent sur ce point, c'est que la matière a précédé le souffle divin qui l'anime [...]. Le livre fondamental de notre foi s'exprime dans ce sens, lorsqu'il nous dépeint les scènes imposantes de la création. Celle-ci n'a été qu'un grand acte de la volonté de Dieu réagissant sur l'inertie de la matière préexistante, et lui intimant la vie et le mouvement 23 .

Ce passage a pu retenir l'attention de Flaubert parmi les lectures auxquelles il se livre dans la période de rédaction de Salammbô. On trouve en effet à la date du 5 août 1860 une lettre à Madame Jules Sandeau où il écrit : «J'entremêle mes lectures puniques (qui ne sont pas légères) d'autres facéties graves. Je me livre maintenant au volumineux bouquin de mon ami le docteur Pouchet sur les générations spontanées 24 . » C'est la nature de cet entrelacement que nous aimerions interroger ici. Il pourrait s'agir d'une récréation savante sans rapport direct avec l'écriture du roman. Mais l'influence des idées de Pouchet, qui ont certainement été connues de Flaubert bien avant la lecture de son grand œuvre (nous savons que FélixArchimède Pouchet était l'élève du père de Flaubert et

19. Saint Jérôme, Euvres complètes, t. III, Louis Vivès, 1878, p. 510. 20. Samuel Cahen, La Bible, traduction nouvelle avec l'hébreu en regard avec des notes philologiques, géographiques et littéraires, Paris, 18311851 (18 vol.), vol. I, p. 1.

21. «Je laboure la Bible de Cahen », écrit Flaubert en juillet 1857 au début de l'élaboration de Salammbô (Correspondance, op. cit., t. II, p. 740, lettre à Ernest Feydeau).

22. Félix-Archimède Pouchet, Hétérogénie ou Traité de la génération spontanée, Paris, Baillière et Fils, 1859, p. 98.

23. Ibid., p. 130.

24. Flaubert, Correspondance, op. cit., t. III, p. 102. 
un ami de la famille) est d'une telle évidence dans la page qui nous intéresse qu'elle engage à une réflexion sur le croisement des « lectures puniques » et de la lecture scientifique.

Félix Pouchet, dans le Traité de la génération spontanée qu'il publie en 1859, défend, contre Pasteur et Milne-Edwards, l'idée d'une «force génésique » qui aurait présidé à la création et qui continuerait de s'exercer dans la formation et la transformation des espèces ${ }^{25}$. Il ne s'agit évidemment pas de comprendre l'hétérogénie comme « la création immédiate d'animaux parfaits, surgissant instantanément de la rencontre fortuite de leurs éléments au milieu de la matière », mais comme une «force encore inexpliquée », une «manifestation plastique » sous l'empire de laquelle «quelques molécules se sont rencontrées pour engendrer un ovule imperceptible, presque un point mathématique ». En somme, "les hétérogénistes se bornent à ne considérer que le point initial de la force vitale et de la matière 26 ». La thèse de Pouchet, qui s'appuie sur une série d'expériences longuement rapportées dans le Traité, se définit comme le prolongement scientifique et moderne de l'intuition antique de la génération spontanée qui s'exprime entre autres dans la philosophie atomiste : «La génération spontanée était presque un dogme pour la plupart des philosophes de l'antiquité27. » Or, ce « dogme » pourrait remonter à la Phénicie, et précisément à la cosmogonie de Sanchoniathon. L'hypothèse est présentée par Renan :

Une théorie de la génération spontanée, caractérisée par les deux mots sacramentels de $\mu \omega \tau$ et de Z $\omega \varphi \alpha \sigma \eta \mu \mathrm{v}$ (ou mieux

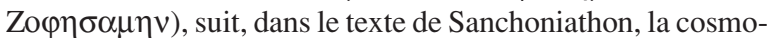
gonie que nous venons d'exposer [...]. M. Ewald rapproche $\mu \omega \tau$ de l'arabe "matière ». M. Bunsen, plus hardi encore, propose de lire Mo $\chi$; ce mot, qu'il explique par l'hébreu, est pour lui identique à M $\omega \chi 0 \varsigma$, qui figure ailleurs comme le nom d'un auteur phénicien, que les Grecs ont pris pour l'inventeur de la philosophie atomistique 28 .

Il existerait donc une pensée originelle, spontanée, des peuples antiques, dont la vérité aurait été perdue au cours de l'ère chrétienne puis retrouvée par les modernes, la cosmogonie orientale pouvant dans ces conditions être lue comme l'expression d'un évolutionnisme primitif.
La version de Flaubert confirme cette interprétation spontanéiste de la cosmogonie phénicienne. L'auteur de Salammbô s'appuie sur la formule de la génération spontanée donnée par l'ensemble des traductions : « c'est d'elle que procéda toute semence de création », et l'infléchit en y intégrant la conception moderne de la continuité de la création : « elle enfermait les germes des choses qui devaient naître ». On relève également dans l'élaboration des versions successives du passage qui donnera « elle enfermait des monstres insensibles, parties incohérentes des formes à naître » (V), l'alternance, pour désigner le même phénomène, de la perspective scientifique et mythique, les « monstres insensibles » pouvant renvoyer à un bestiaire mythique et les «parties incohérentes » à un énoncé pseudoscientifique. La première version donnait « animaux inscients» (pour ce qui deviendra «monstres insensibles ») et «germes des choses» (pour «parties incohérentes »). «Inscients » appartient à un lexique philosophique et moderne ; quant à «germes », il a la particularité d'être à la fois «ancien » et « moderne »- le sens du mot étant en cours de spécialisation au XIX ${ }^{\mathrm{e}}$ siècle. La recherche d'une articulation entre le mythe et la pensée scientifique s'observe également dans les versions 1,2 et 3 (IV) quand il s'agit d'expliquer la formation de la terre en termes physiques : «mouvement de rotation » et «sa propre pesanteur le brisa ». On peut parler ici d'une tentative pour formuler le «newtonisme phénicien » sur la base d'une terminologie moderne qui sert de support à l'image. C'est le mouvement qui crée la terre mais aussi les dieux, soumis comme elle aux lois de la physique que les anciens ne pouvaient exprimer de manière scientifique mais dont ils percevaient la vérité. Flaubert s'abstient finalement d'écrire « rotation » ou «pesanteur» pour les dire autrement en les retraduisant à rebours, en reconstituant par l'écriture la pauvreté lexicale et la richesse métaphorique du texte ancien.

\footnotetext{
25. Pouchet, Hétérogénie ou Traité..., op. cit., p. 8-9.

26. Ibid., p. 347.

27. Ibid., p. 11.

28. Renan, Sanchoniathon, op. cit., p. 15.
} 
Derrière la « querelle de Sanchoniathon » on découvre donc une autre bataille, celle de la génération spontanée, autrement virulente et dont le retentissement touche un public beaucoup plus large, mais très fortement liée à la première. La question de la Genèse se pose à la fois, nous l'avons vu, en termes philologiques, religieux et scientifiques. Les trois niveaux de réflexion sont pensés ensemble : c'est encore le cas au XIX ${ }^{\mathrm{e}}$ siècle. La polémique scientifique ne se sépare jamais du combat religieux ou antireligieux. Toucher à la Genèse, s'en emparer et la récrire en la retournant comme l'a fait Flaubert pour Salammbô, c'est toucher au manteau de Tanit : il ne s'agit ni d'un exercice de style ni d'une «folie » archéologique mais d'un geste qui engage et dont les enjeux sont très clairs dans ce moment de grande violence idéologique qui marque la France du Second Empire. L'Académie des Sciences met à l'index l'ouvrage de Pouchet en condamnant « cette monstruosité philosophique, cette idée destinée à fournir des armes au panthéisme et au matérialisme, à saper les bases de la religion et de 1'État 29 ». Quelques mois plus tard éclatera la «querelle de Salammbô », au cours de laquelle on accusera Flaubert de vouloir « ramener le paganisme 30 ». La mauvaise foi et l'esprit de parti sont des révélateurs très sûrs du sentiment de danger que peut susciter une œuvre. Oui, la menace que Salammbô fait porter sur « les bases de la religion » est bien réelle : s'il n'est pas question, du moins pas en ces termes, de « ramener le paganisme », le roman, en s'appuyant sur la Bible et en la détournant, inévitablement la questionne, et pas seulement à l'échelle du court texte dont nous avons présenté la « genèse ».

29. Rapporté par Jean Bruneau dans Flaubert, Correspondance, éd. cit., t. III, p. 1096. Flaubert le rappellera dans le Dictionnaire des idées reçues: " Génération spontanée : théorie de socialiste ».

30. Ibid., t. III, p. 313, lettre de Flaubert à Jules Duplan : « À propos d'attaque, sais-tu que j'ai été dénoncé comme corrupteur des mœurs dans deux églises ? $1^{\circ}$ église Sainte-Clotilde, $2^{\circ}$ église de la Trinité (rue de Clichy). Là, le prédicateur s'appelait l'abbé Becel. J'ignore le nom de l'autre. Tous deux ont tonné contre l'impudicité des mascarades, contre le costume de Salammbô ! Ledit Becel a rappelé la Bovary et prétend que cette fois je veux ramener le paganisme. Ainsi l'Académie et le clergé m'exècrent. Ça me flatte, et ça m'excite !!! » 
AGNÈs Bouvier est agrégée de Lettres modernes et docteur de l'université Paris VIII. Membre de l'équipe « Flaubert » de l'ITEM et du comité de rédaction de la revue Flaubert. Revue critique et génétique, elle participe au projet FRACTAL (Flaubert-ReligionsAntiquité-Intertexte Franco-Allemand) en partenariat avec l'université de Munich. Elle a publié des articles sur Flaubert et sur les religions au XIXe siècle, parus notamment dans French Studies et Romantisme. Elle collabore au dictionnaire « Flaubert » à paraitre aux éditions Champion.

Agnès Bouvier, agnes.bouvier@gmail.com

\section{La « Genèse » de Salammbô}

L'insertion dans Salammbô de la traduction/adaptation d'un récit de genèse issu des fragments d'une Histoire phénicienne de Philon, amène à s'interroger globalement sur l'œuvre comme résurrection fictive d'un monde et d'une langue perdus qui menace la charge symbolique du texte biblique dans la tradition occidentale. La comparaison de la traduction de Flaubert avec plusieurs traductions contemporaines met en évidence sa volonté de retrouver le caractère énigmatique et étranger au rationalisme occidental de l'original perdu, de restaurer - et de repenser - une langue épousant la dynamique du vivant et de la matière. Du même coup, le texte biblique de la Genèse se trouve lui-même dénoncé implicitement comme détournement et avatar dérisoire d'un original matérialiste, et son autorité est sourdement attaquée par le roman flaubertien et la manière dont il ordonne en strates poétiques la série historique des discours. De ce point de vue, Salammbô intériorise et approfondit le travail de déconstruction des textes sacrés par les Lumières et représente une version poétique radicale de la modernité (dont le retour à l'origine est la clé paradoxale) comme mise en cause des traditions judéo-chrétiennes.

The insertion in Salammbô of the translation/adaptation of a genesis narrative derived from fragments of Philon's Phoenician History leads us to globally question the work as a fictional resurrection of a world and a language that have been lost, as it threatens the symbolic weight of the biblical text in the Western tradition. The comparison of Flaubert's translation with several contemporary ones underscores his ambition to recover the original's enigmatic character that is foreign to Western rationalism, and restore, as well as reconsider, a language that adopts the dynamics of the living being and matter. At the same time the Genesis as a biblical text is itself implicitly denounced as an appropriation and a trivial transformation of a materialist original and its authority is underhandedly attacked by the Flaubertian novel as it organizes the historical discourses in poetic strata. From this standpoint Salammbô internalizes and develops the Age of Enlightenment's deconstruction of sacred texts. Thus it presents a radical poetic version of modernity (whose return to the origin is the paradoxical key) as a challenging of Judeo-Christian traditions.

Dass Flaubert in seinen Roman Salammbô eine Übersetzung/Adaption einer Schöpfungsgeschichte einfügt, die Fragmenten einer Histoire phénicienne von Philon entstammt, wirft generell die Frage nach dem Werk als fiktionale Wiedererweckung einer verlorenen Welt und ihrer Sprache auf, die den Symbolgehalt des biblischen Textes in der abendländischen Tradition bedroht. Der Vergleich seiner Übersetzung mit verschiedenen zeitgleichen Übersetzungen lässt das Bestreben Flauberts deutlich werden, den rätselhaften wie auch dem abendländischen Rationalismus fremden Charakter des verlorenen Originals wiederzufinden, eine Sprache wiederherzustellen - und neu zu denken -, die mit der Dynamik des Lebendigen und der Materie im Einklang ist. Damit wird der biblische Text der Genesis implizit als Verfremdung und armselige Anverwandlung eines materialistischen Originals bloßgestellt; seine Autorität wird durch Flauberts Roman und seine Art und Weise, die historische Abfolge der Diskurse in poetischen Schichten aufzutragen, heimlich untergraben. Aus dieser Perspektive macht sich Salammbô die Dekonstruktion heiliger Texte zu eigen, die in der Aufklärung wurzelt, vertieft sie und stellt zugleich eine radikale poetische Spielart der Moderne als Infragestellung der jüdisch-christlichen Traditionen dar, deren paradoxaler Schlüssel in ihrer Rückkehr zum Ursprung liegt.
La inserción, en Salammbô, de la traducción/adaptación de un relato de génesis tomado de los fragmentos de una Historia fenicia de Filón, plantea un interrogante global sobre la obra como resurrección ficticia de una lengua y un mundo perdidos que afecta la carga simbólica del texto bíblico en la tradición occidental. La comparación de la traducción de Flaubert con varias traducciones contemporáneas pone en evidencia su deseo de recuperar el carácter enigmático y ajeno al racionalismo occidental del original perdido, de restaurar-y reconsiderar- una lengua acorde a la dinámica de lo viviente y de la materia. Al mismo tiempo, el texto bíblico mismo de la Génesis es acusado implícitamente de desvío y avatar irrisorio de un original materialista, y su autoridad es atacada veladamente por la novela flaubertiana, así como la manera de ordenar en estratos poéticos la serie histórica de los discursos. En esta perspectiva, Salammbô interioriza y profundiza el trabajo de deconstrucción de los textos sagrados por parte de la Ilustración y representa una versión poética radical de la modernidad (en la que la vuelta a los orígenes es la clave paradójica) en tanto que cuestionamiento de las tradiciones judeocristianas.

A inserção em Salammbô da tradução/adaptação de uma narrativa de génese procedente de fragmentos de uma Histoire phénicienne de Fílon permite interrogar globalmente a obra como ressurreição fictícia de um mundo e de uma língua perdidos, ameaçadores para a carga simbólica do texto bíblico na tradição ocidental. A comparação da tradução de Flaubert com várias traduções contemporâneas destaca a sua vontade de recuperar o carácter enigmático e estranho ao racionalismo ocidental do original perdido, e de restaurar - e repensar - uma língua que se casa com a dinâmica da vida e da matéria. Ao mesmo tempo, o texto bíblico da Génese é implicitamente denunciado como desvio e sucedâneo irrisório de um original matérialista, e a sua autoridade é atacada em surdina pelo romance flaubertiano e pelo modo como nele se ordena em estratos poéticos a série histórica dos discursos. Deste ponto de vista, Salammbô interioriza e aprofunda o trabalho de desconstrução iluminista dos textos sagrados e representa uma versão poética radical da modernidade, que tem o regresso às origens como chave paradoxal e que põe em causa as tradições judeocristãs.

L'inserimento in Salammbô di una parte di traduzione e adattamento di un racconto di genesi tratto dai frammenti di una Histoire phénicienne di Filone induce a interrogarsi sull'opera come resurrezione fittizia di un mondo e di una lingua perduti che minaccia la carica simbolica del testo biblico nella tradizione occidentale. La comparazione della traduzione di Flaubert con altre traduzioni coeve mette in luce la sua volontà di ritrovare il carattere enigmatico e estraneo al razionalismo occidentale dell'originale perduto, di restaurare - e ripensare - una lingua che sposi la dinamica del vivente e della materia. Allo stesso tempo, il testo biblico del Genesi si trova anch'esso denunciato implicitamente come diversione e trasformazione derisoria di un originale materialista, e la sua autorità è attaccata sordamente dal romanzo flaubertiano e dalla maniera con la quale ordina in strati poetici la serie storica dei discorsi. Da questo punto di vista, Salammbô interiorizza e approfondisce il lavoro di decostruzione dei testi sacri già svolto dall'Illuminismo e rappresenta una versione poetica radicale della modernità (della quale il ritorno all'origine è la chiave paradossale) come messa in discussione delle tradizioni giudeo-cristiane. 